\title{
4. Das Gerbertsche Chanson-Fragment des frühen 15. Jahrhunderts aus der ehemaligen Bibliothek des Klosters St. Georgen in Villingen
}

1. Martin Gerbert, Fürstabt von St. Blasien, hat in seinem magistralen musikhistorischen Werk De Cantu et Musica Sacra von 1774 eine ganze Zahl von „,nachgestochenen“ Facsimile-Abbildungen aus mittelalterlichen $\mathrm{Mu}-$ sikhandschriften publiziert. Dazu gehört ein dem frühen 15. Jahrhundert zugehörendes schwarz notiertes Virelai mit dem Textbeginn Mais qu'il vous viengne $^{1}$ (vgl. Abb. 4a)); es ist in Gerberts Abbildung in einer Fassung zu drei Stimmen wiedergegeben, wogegen die einzige zuverlässig vergleichbare Konkordanz dieser Chanson im norditalienischen, aber viele französische Chansonsätze enthaltenden Codex Reina (= Paris 6771) nur zwei Stimmen, ohne den CT Gerberts, bietet. Nach dem Gerbertschen Facsimile von 1774 hat wiederum Raphael Georg Kiesewetter im Jahre 1834 ein Facsimile der Chansonnotation sowie erstmals eine Partitur dieses in moderne Notation übertragenen Satzes publiziert, wie sie ihm ein „,in der musikalischen Paläologie ungemein bewanderter Freund zugesendet" hatte; ${ }^{2}$ wer dieser Freund war, ließ sich nicht eruieren.

K: - Paris 6771, fol. 77; anon.

- Groningen 70 (Tabulatur), fol. I.2'; Kf.: Empris domoyrs, anon.

- Augsburg 38, fol. 173'-180'/ Philadelphia 36, fol. 207-216’; „Mais quil vous vierge“" (Traktatzitat); anon.

Zur „Leistungsfähigkeit“ dieser Konkordanzen seien bereits hier einige Erläuterungen angeschlossen. Ein Kommentar zum bekannten und gut

1 Vgl. Martin Gerbert, De Cantu et Musica Sacra a prima ecclesiae aetate usque ad praesens tempus, St. Blasien 1774, Bd. 2, Tab. XIX, mit Textbeginn in der Schreibung Mays quil wus viengne ... .

2 Raphael Georg Kiesewetter, Geschichte der europäisch-abendländischen oder unserer heutigen Musik ... , Leipzig 1834: „Facsimile“ auf p. IV, No. 3, Übertragung davon auf p. V; das Zitat S. 41. - Eine neue Edition des Satzes nach Gerberts Abbildung bietet Polyphonic Music of the Fourteenth Century ... (= French Secular Music ..., vol. 21, ... Edited by Gordon K. Green), Monaco 1987, S. 102f., Nr. 43, sowie S. 175, Nr. 43. 
untersuchten Codex Reina erscheint nicht nötig. ${ }^{3}$ Die zweite, nicht ganz vollständige Konkordanz, ebenfalls nur zweistimmig und mit abweichender Textmarke, liegt in einem frühen Fragment einer Klaviertabulatur des späten 14. Jahrhunderts vor, das in die Inkunabel der Groninger Universitätsbibliothek Inc. no. 70 eingebunden worden ist. ${ }^{4}$ Der Satz ist gegenüber den beiden anderen Fassungen um eine Sekunde höher notiert. Kurz vor dem Groninger Fragmentenschluß, also in den Takten 20-23 der dem in Anm. 4 genannten Aufsatz beigegebenen Übertragung, scheint der Notentext des Tabulaturfragments, vielleicht schon derjenige der intavolierten Vorlage, gestört. Die Notation der Tabulatur zeigt französische und italienische Einflüsse; eine Provenienz des 1477 in Groningen in den genannten Inkunabeleinband eingearbeiteten Blattes ist darüberhinaus allerdings nicht sicher. Die Kolorierung der rechten Spielhand, meist bei längeren Notenwerten der vokalen Vorlage, behindert eine Auswertung der Intavolierung freilich sehr, vor allem dann, wenn diese mit den beiden vokalen Chansonfassungen textkritisch verglichen und beurteilt werden soll; deshalb wird sie eben dazu im Folgenden nicht im Einzelnen herangezogen. Und schließlich findet sich der Textbeginn der Chanson in einem anonymen süddeutschen Traktat dieser Zeit, der in zwei Handschriften überliefert ist und Formen kompositorischer Gattungen beschreibt; der vorliegende Satz ist darin als Beispiel der Form des Virelai zitiert, nicht jedoch musikalisch wiedergegeben. ${ }^{5}$

Zurück zum Gerbertschen Beleg. Über die Herkunft der 1774 „Ex Msc. Scheda Monasterii S. Georgii" "6 reproduzierten Manuskriptvorlage dieses Virelais gibt Gerbert selber, in der neueren Musikforschung häufig übersehen, weitere und ausführlichere Auskunft: ,... damus ... exemplum aliquod ex membrana itidem, quae compacturae serviebat libri choralis monasterii monialium Urspringensium, nunc vero servatur in monasterio $S$.

3 Vgl. Carola Hertel, Chansonvertonungen des 14. Jahrhunderts in Norditalien. Untersuchungen zum Überlieferungsbestand des Codex Reina, Hildesheim etc. 2002 (= Musikwissenschaftliche Publikationen, Bd. 17), bes. S. 17ff; direkt zur Gerbertschen Quelle äußert sich diese Arbeit nicht.

4 Vgl. Maria van Daalen/Frank Harrison, Two keyboard Intabulations of the late fourteenth century on a manuscript leaf now in the Netherlands, TVNM 34 (1984), S. 97-108. - Der hier mehrfach gebotene Hinweis auf eine weitere zweistimmige Konkordanz in Prag, Universitätsbibliothek, Ms. XI. E. 9, fol. 77, ist irrig; zugrunde liegt offenbar die falsche Auflösung der in der Sekundärliteratur üblichen Handschriftensigle „PR“ (für „Paris, Reina“) als „Prag“ o. ä.

5 Vgl. dazu und zum Folgenden Martin Staehelin, Beschreibungen und Beispiele musikalischer Formen in einem unbeachteten Traktat des frühen 15. Jahrhunderts, AfMw 31 (1974), S. 237-242; vgl. dazu unten, Anm. 18.

6 So die Überschrift über dem Gerbertschen Abdruck; vgl. oben, Anm. 1. 
Georgii, quod est Villingae, continetque contrapunctum cancionis Gallicae“.7 Es handelte sich also um ein Pergamentblatt, das in den Einband eines Choralbuches aus dem Benediktinerinnen-Kloster Urspring eingearbeitet war und sich 1774 im Kloster St. Georgen in Villingen (im Schwarzwald) befand. Kenntnis von dem Urspringer Codex und damit auch dem Chanson-Blatt könnte Gerbert allerdings schon 1761 in St. Georgen bekommen haben, denn im Mai dieses Jahres nutzte er für sein damals in Arbeit befindliches musik- und liturgiehistorisches Werk De Cantu et Musica Sacra an Ort und Stelle jene Manuskriptbestände, die Coelestin Wahl, der Abt von St. Georgen, nach dem reformationsbedingten Niedergang seines Klosters dort zusammengebracht hatte. Von Abt Coelestin sprach Gerbert ungemein lobend, ,qui subsidia litterarum omnem in modum auget, non novis tantum doctis libris ..., verum etiam antiquis codicibus ...: licuitque mihi, non pauca ad rem liturgicam annotare. ... Vix quidem illi, paucis exceptis, saeculum XV. antecedunt, multa tamen ... continent singularia“. 8

Natürlich ist das fragliche Fragment - und um ein Fragment muß es sich gehandelt haben, dazu unten mehr - der Aufmerksamkeit und dem Interesse eines so gelehrten Quellenforschers wie Friedrich Ludwig nicht entgangen. Seine Recherchen nach dem Verbleib des Bruchstücks waren erfolglos: in dem Material aus St. Georgen, das 1807 nach Karlsruhe gelangte, ließ sich das Blatt jedenfalls nicht finden. ${ }^{9}$ Bemühungen späterer Musikhistoriker - selbst noch nach 1950 - , das originale Fragment anderswo aufzuspüren, fehlten nicht, blieben aber, weil ebenfalls erfolglos, in der musikhistorischen Literatur unerwähnt; mündliche Nachfragen nach dem Schicksal des Blattes unter Kollegen bewiesen allerdings ein nicht geringes Interesse daran, das Bruchstück im Original vielleicht doch wieder ans Licht gefördert zu sehen. Die Möglichkeit, daß es bereits im verheerenden Klosterbrand von St. Blasien von 1768 zerstört worden wäre und eben deshalb unauffindbar geblieben sei, scheidet deshalb aus, weil Gerbert das Fragment sechs Jahre nach jenem Brand ja ausdrücklich als in St. Georgen vorhanden bezeichnete. Jenen umfangreichen, auch musiktheoretischen Sammelband dagegen, der ausdrücklich „Bibliothecae San-Blasianae“ gehörte und verschiedene handschriftliche Traktate von Boethius, Guido

7 Gerbert (s. oben, Anm. 1), Bd. 2, S. 63.

8 Martin Gerbert, Iter Alemannicum, accedit Italicum et Gallicum ..., St. Blasien 1765, S. 298f. Es sei an dieser Stelle auch festgehalten, daß die neueren mehrbändigen Ausgaben der Gerbertschen Korrespondenz (Karlsruhe 1931ff.) sowie der Gerbertschen Briefe und Akten (Karlsruhe 1957ff.) keinerlei Information über das hier behandelte Chansonfragment bringen.

9 Vgl. Friedrich Ludwig [Hrsgr.]: Guillaume de Machaut, Musikalische Werke, Bd. 2, Leipzig 1928 (= PäM 3,1), S. 42*. 
von Arezzo, Berno, Wilhelm von Hirsau, Odo und anderen Autoren enthielt und im Brand von 1768 tatsächlich zerstört wurde, beklagte Gerbert im gleichen Werk, in dem er das Virelai-Fragment nannte, nachdrücklich als großen Verlust. ${ }^{10}$

Auch erneute, im Zuge dieser Darlegung unternommene Bemühungen, das Bruchstück oder den genannten choralen Trägerband aus Urspring wiederaufzufinden, sind erfolglos geblieben: unter den wenigen bekannten, aus St. Georgen erhaltenen Manuskriptresten fand sich auch jetzt keine weiterführende Spur, ${ }^{11}$ und über die alten Bibliotheksbestände von Urspring und deren Verbleib fehlen Informationen fast ganz. ${ }^{12}$ Die Ungewiß-

10 Gerbert (wie oben, Anm. 1), St. Blasien 1774, Bd. 1, praefatio; ergänzend Friedrich Nicolai, Beschreibung einer Reise durch Deutschland und die Schweiz im Jahre 1781, Bd. 12, Berlin/Stettin 1796, S. 71f. Aus dem Inhalt des erwähnten Theoretikercodex edierte Gerbert in seinen Scriptores ecclesiastici de musica sacra potissimum, St. Blasien 1784, eine ganze Zahl von musiktheoretischen Traktaten. Daß es zweifellos eben diese Handschrift war, deren Verlust Gerbert so sehr bedauerte, und nicht wie gelegentlich zu lesen ist - das heute in der Londoner British Library befindliche, von Gerbert (s. oben, Anm. 1), Bd. 1, S. 376f., behandelte Ms. Add. 27630 [= LoD] mit älterem Organal- und Motettenrepertoire, sei hier ausdrücklich festgehalten; vgl. dessen Ausgabe im EdM, Bd. 52/53, hrsg. von Wolfgang Dömling, Kassel etc. 1972.

11 Es wurden Auskünfte bei den folgenden Institutionen eingeholt: Freiburg i. Br., Universitätsarchiv und Universitätsbibliothek (Dr. Angela Karasch); Karlsruhe, Badische Landesbibliothek (Rainer Fürst) und Generallandesarchiv (Dr. Jutta Krimm-Beumann); Rastatt, Historische Lehrerbibliothek des Ludwig-WilhelmGymnasiums (Hans Heid); Stiftsarchiv St. Paul im Lavanttal (Dr. Rudolf Freisitzer); Stadtarchiv Villingen-Schwenningen (Dr. Heinrich Maulhardt). Den vorstehend in Klammern genannten Damen und Herren sei für ihre freundlichen Auskünfte gedankt. - Vgl. insgesamt Gerhard Stamm, Zur Geschichte der Bibliothek [von St. Blasien], in: Das tausendjährige St. Blasien, ... Ausstellung ... 1983, Karlsruhe 1983, Bd. 2, S. 171-200.

12 Vgl. Immo Eberl, Geschichte des Benediktinerinnenklosters Urspring bei Schelklingen 1127-1806, Stuttgart 1978 (= Schriften zur südwestdeutschen Landeskunde, Bd. 13), bes. S. 297 (zur Bibliotheksgeschichte) und S. 59-67 (zur Bindung von Urspring an St. Georgen). Von Immo Eberl, Regesten zur Geschichte des Benediktinerinnenklosters Urspring bei Schelklingen 1127-1806, Stuttgart 1978 (= Schriften zur Südwestdeutschen Landeskunde, Bd. 14), S. 213, Nr. 443, wird eine Bücherschenkung des Priesters Eberhard Wirtschafft, Kaplans der Herren von Ellerbach, an den Urspringer Konvent im Jahre 1441 erwähnt; etwa gleichzeitig stiftete auch Walther von Freyberg 18 Bücher - Näheres bleibt in beiden Fällen unbekannt. Josef Ludwig Fischer, Entwicklungsgeschichte des Benediktinerinnenstiftes Urspring, Studien und Mitteilungen zur Geschichte des Benediktinerordens und seiner Zweige 38 (1917), S. 199-234, und 39 (1918), S. 45-67, bietet zur Urspringer Bibliotheksgeschichte nur (39 (1918), S. 48) die Mitteilung, daß die 1577 verstorbene Nonne Anna von Neuneck mit ihrer Mit-Konventualin Katharina von Westerstetten ,sechs große Choralbücher auf Pergament zierlich geschrieben“ habe. 
heit vergrößert sich dadurch, daß nicht sicher ist, ob der gesuchte Choralband nach Gerberts entsprechender Nennung und gedruckter Auswertung im Jahre 1774 weiterhin im Kloster St. Georgen verblieb oder nach Urspring zurückging; die Verbindungen von St. Georgen und Urspring waren seit alters eng.

2. Etwas Weniges und bisher Übersehenes zum Original des fraglichen Fragmentes - dessen Niederschrift man von seinem Bild her ins frühe 15. Jahrhundert setzen möchte - kann die Gerbertsche Reproduktion allerdings gleichwohl lehren. Es betrifft dies einmal die Tatsache, daß kurz vor dem Ende des T, dabei am rechten Rand des Schriftfeldes, der Rest des Notensystems leer geblieben ist; die letzten sieben Noten nach dieser musikalischen Lücke folgen erst am Beginn des nächsten und damit letzten TNotensystems. Diese von Kiesewetter zwar bemerkte, aber nicht weiter erklärte Textlücke von fast drei tempora ist wohl am einleuchtendsten mit einer buchbinderischen Beeinträchtigung des Pergamentrandes an dieser Stelle zu begründen, wahrscheinlich verursacht durch einen seitlichen Einschnitt, der dort für die quer verlaufenden Schnüre eines Rückenbundes Platz schaffen sollte; daß in Gerberts Reproduktion die fünf Notenlinien dieses Systems bis ganz an den rechten Rand ausgezogen worden sind, kann diese Vermutung nicht entkräften. ${ }^{13}$ - Dieselbe vermutete Beschädigung des seitlichen Blattrandes durch den Buchbinder kann auch die Fragmentform des Blattes bekräftigen: dieses war, da ja buchbinderisch verarbeitet, schon damals nicht ein originales Einzelblatt mit musikalischer Notation, sondern ein aus dem Zusammenhang einer pergamentenen und auf sauberes Bild angelegten eigentlichen Musikhandschrift mit Mehrstim-

Im vorliegenden Zusammenhang hilft das auch nicht weiter. - Herrn Kollegen Immo Eberl sei auch hier für freundlich gewährte Hinweise gedankt.

13 Das gilt schon deshalb, weil die Wiedergabe des Notenbilds bei Gerbert derart präzise und überall übereinstimmend gleiche Systeme nachbildet, daß hier ein sehr genau und mit den neueren Mitteln des Plattenstichs des 18. Jahrhunderts arbeitender Stecher tätig gewesen sein muß. Entsprechendes gilt für die Notenköpfe und -cauden, die in einem Manuskript der Zeit vor 1400 niemals so übereinstimmend gleichförmig ausgefallen wären. Nach den von Green (s. oben, Anm. 2), p. XVI, benannten musikalischen Fehlern in Gerberts Abbildung erscheint allerdings denkbar, daß auch nicht deren originale Vorlage, sondern deren Nachstecher, der Mensuralnotation und der Sprache unkundig, für jene Fehler verantwortlich war. Übrigens hat Kiesewetter (s. oben, Anm. 2) in seinem an Gerberts Vorlage orientierten „Facsimile“ die bei Gerbert am Beginn des letzten T-Systems stehenden sieben Schluß-Noten ohne weitere Bemerkung in die erwähnte Textlücke am rechten Rand des vorangegangenen Systems zurückverschoben, so daß das im Original außen rechts entleerte System bei Kiesewetter vollständig gefüllt ist und das bei Gerbert dann noch folgende sechste System stillschweigend wegfällt. 
migkeit isoliertes Bruchstück. Für die ursprüngliche Herkunft aus einem größeren Manuskriptkomplex spricht jedoch der ausgeglichen unterlegte C-Worttext, der sich damit auffällig geordneter präsentiert, als man es in bloß versprengten, zumal deutschen Einzelnotationen des 15. Jahrhunderts häufig findet. Als ganze ist die ursprüngliche Gesamthandschrift freilich mit Sicherheit verloren; über ihren Umfang und Inhalt läßt sich heute nichts mehr sagen, auch nicht vom Gerbertschen Fragment her.

Wichtiger erscheint jedoch das, was das Notierte über die Provenienz des Blattes auszusagen vermag. Der französische Text, den der gute und erfahrene Paläographe Gerbert der C-Stimme offenbar vorlagengetreu unterlegte, ist nach seinen regionalen Eigenheiten ostfranzösischer Herkunft: sie deuten auf Wallonien oder Lothringen. ${ }^{14}$ Es ist zunächst nicht ausgeschlossen, daß er bzw. die in Gerberts Fragment kopierte Vorlage auch dort entstanden sein und in früher Zeit den Weg in das Kloster Urspring gefunden haben könnte. Gegen diese Möglichkeit spricht allerdings entschieden die am Anfang des T gegebene Stimmbezeichnung „Tenor huius“: diese Art, eine besondere Stimme als zu einer bestimmten Komposition gehörig $\mathrm{zu}$ bezeichnen, tritt in deutschen und dem zentralen Europa entstammenden Handschriften mit mehrstimmiger Musik seit dem Anfang des 15. Jahrhunderts nicht selten auf, ${ }^{15}$ während sie, soweit zu erkennen, in origi-

14 Herrn Kollegen Ulrich Mölk, Göttingen, sei auch hier für sehr hilfreiche Beratung in dichtungs-, sprachgeschichtlicher und -geographischer Hinsicht gedankt. Ich gebe seine Mitteilungen gerne im Folgenden weiter, teilweise durch eigene Angaben ergänzt. Zunächst ist die hier vertonte Strophe ein metrisch, mithin nach Silbenzahl und Reim, korrekt gebauter Siebenzeiler der Struktur 7a', 7b, 7b, 7a' / 7c, 7c, 7a'. Sprachlich ist sie in der Gerbertschen Fassung, wie erwähnt, ostfranzösischer Herkunft (Wallonien, Lothringen), während die Version im Codex Reina in Kontaktgebiet Norditalien (Padua?) geschrieben sein dürfte; vgl. dazu Hertel (s. oben, Anm. 3).

Die Textherstellung nach der Gerbertschen Fassung ergibt: „,[1] Mais qu'il wus viengne a plaisanche, [2] dame, en porcas de pitey, [3] donés moy par carité [4] de ma dolor aliantche. [5] Car del doulz bien, a mon tens, [6] sui si pores diferens [7] que de mort sui en doutanche." Dazu Textkritisches: [4] dalor - [5] der; doulr [!] - [6] sin; pores (regionale Variante von povres) - [7] e; dautanche.

Übersetzung ins Deutsche ,Wofern es Euch gefällt, Dame, um mir Mitleid zu erweisen [wörtlich: im Verfolgen von Mitleid], gebt mir aus Barmherzigkeit Erleichterung von meinem Schmerz; denn in Bezug auf die süße Freude bin ich, zeit meines Lebens, ein so armseliger Anderer [ $=$ von ... so weit entfernt], daß ich vor dem Tode in Angst bin“.

15 Für ein einzelnes, aber diese Stimmzuweisung besonders häufig nutzendes Beispiel (übrigens auch für andere Stimmen als nur den T) sei auf die umfangreichen Fragmente einer offenbar in Breslau etwa um 1450 geschriebenen Handschrift mit Mehrstimmigkeit hingewiesen: KÜ III; vgl. auch KÜ VIII, S. 258 und 260. 
när französischen und, wie der Codex Reina zeigt, auch norditalienischen Manuskripten dieser Zeit fehlt.

Daraus ergeben sich für das von Gerbert wiedergegebene originale Blatt zwei Möglichkeiten: nach der einen könnte das Fragment in einem sprachlichen „Kontaktgebiet“ entstanden sein, in dem sich französische und deutsche Sprache und Sprachkenntnisse berührt oder gar überdeckt hätten: das würde die Herkunft in einem Gebiet etwa nördlich des Elsaß über die Regionen um Metz und Luxemburg bis gegen Lüttich verlaufend annehmen lassen.

Die andere, nach dem Folgenden jedoch insgesamt wahrscheinlichere Möglichkeit wäre, daß das Blatt von einem, selbst bei einem französischen Worttext um Kopiertreue wenigstens bemühten deutschen Kopisten vielleicht sogar in der näheren Umgebung von Urspring - beschrieben, dabei zugleich mit dem genannten Vermerk „Tenor huius“ versehen worden und so in dieses Kloster gelangt wäre. Dafür spricht, daß der Worttext einige fehlerhafte Lesarten zeigt, die man einem muttersprachlich französischen Schreiber kaum wird zumuten können. ${ }^{16}$ Angesichts der beiden nur zweistimmigen Konkordanzen des Virelai im Codex Reina und im Groninger Tabulaturfragment ${ }^{17}$ ist es sodann, wenngleich nicht gesichert, so auch nicht ausgeschlossen, daß jene Lesarten ebenfalls eine deutsche Herkunft des Gerbertschen Bruchstückes profilieren: das Fehlen des CT in der Version des italienisch-französisch orientierten Codex Reina, vielleicht auch in derjenigen der Groninger Intavolierung, scheint die dreistimmige Überlieferung im Gerbertschen Fragment deutlich abzusondern, und vielleicht ist diese Dreistimmigkeit sogar das Ergebnis einer erst in deutschem Gebiet erfolgten Erweiterung des Satzes.

Für eine deutsche Provenienz - im Blick auf Urspring nun tatsächlich eine solche aus süddeutsch-bayerischem Gebiet - könnte schließlich ebenfalls die Verbindung sprechen, in der das Fragment mit dem oben erwähnten kleinen anonymen Musiktraktat steht, dessen eine Handschrift $(\mathrm{P})$ aus süddeutschem oder österreichischem, dessen andere Handschrift $(\mathrm{H})$ offenbar aus süddeutsch-schwäbischem Gebiet stammt, ${ }^{18}$ nach ihrem Besitzer-

16 Vgl. oben, Anm. 14: Fast alle dort zu [4-7] aufgeführten Schreibungen sind eben solche fehlerhaften Lesarten. - Der am Ende dieser Ausführungen gegebene Hinweis auf den unter den Konkordanzen aufgeführten anonymen Musiktraktat und der dort gebotene Titel „Mais quil vous vierge“ [sic] läßt sogar darüber spekulieren, ob vielleicht eine Nonne von Urspring, die wenigstens geistlicher französischer Begriffe kundig war, das Fragment geschrieben haben könnte.

17 Vgl. oben, Anm. 4.

18 Vgl. oben, Anm. 5. 1974 wurden für die Besitzerbibliotheken in „Philadelphia“ und „Harburg“ die Siglen P und H gewählt und so auch im textkritischen Apparat je- 
vermerk im 17. Jahrhundert der Benediktinerabteil Hl. Kreuz in Donauwörth gehörte und möglicherweise auch dort entstanden war. Beide Manuskripte gehen nach der Kollation ihrer Lesarten mit hoher Wahrscheinlichkeit auf dieselbe, heute verlorene Vorlage zurück, und beide Codices zeigen, mit Rücksicht auf das Benediktinerinnenkloster Urspring kennzeichnend genug, Spuren der Entstehung im Bereich des Benediktinerordens.

Der erwähnte anonyme Traktat bietet Beschreibungen mehrstimmiger musikalischer, meist weltlicher Formen, wie sie um 1400 im Schwange waren. Die zu deren anschaulicher Illustration beigegebenen Text-Incipits von konkreten Form-Beispielen bekräftigen die genannte Datierung für die Entstehung des Traktattextes ohne weiteres, auch wenn beide Handschriften, also $\mathrm{P}$ und $\mathrm{H}$, einige Jahrzehnte jünger sein dürften. In dem Traktattext erscheint nun zur formalen Beschreibung eines Virelais tatsächlich der Verweis auf ein „exemplum“ mit dem Textincipit Mais quil vous vierge; dieses Incipit scheint die Präsenz des kompositorischen Textes des Virelai im genannten süddeutschen Gebiet zu bestätigen. Und diesen geographischen Bereich dürfte auch die - ohnehin nicht übermäßig große - Distanz zwischen Urspring und Donauwörth nicht in Frage stellen.

ner Veröffentlichung genutzt; mittlerweile ist $\mathrm{H}$, zusammen mit manchen anderen Handschriften aus Oettingen-Wallersteinschem Besitz, an die Universitätsbibliothek Augsburg übergegangen, wo die Handschrift weiterhin die (vorher gültige) Signatur Cod. II, 1, 2 38 trägt; freundliche Auskunft von Herrn Günther Grünsteudel M.A. von der Universitätsbibliothek Augsburg. 